\title{
Prevalence and prognostic value of increased uptake in bone SPECT/CT in asymptomatic wrists
}

Maresa Allgayer ${ }^{1,3^{*}}$, Urs Hug $^{2}$, Justus Egidius Roos ${ }^{1}$, Maria del Sol Pérez Lago ${ }^{1}$, Damian Wild ${ }^{3}$, Dirk Lehnick ${ }^{4}$ and Klaus Strobel ${ }^{1}$

\author{
* Correspondence: \\ maresa.allgayer@unibas.ch \\ ${ }^{1}$ Nuclear Medicine and Radiology, \\ Cantonal Hospital Lucerne, \\ Spitalstrasse, 6000 Luzern 16, \\ Switzerland \\ ${ }^{3}$ Clinic of Radiology and Nuclear \\ Medicine, University Hospital Basel, \\ Spitalstrasse 21, 4056 Basel, \\ Switzerland \\ Full list of author information is \\ available at the end of the article
}

\begin{abstract}
Purpose: To evaluate the prevalence and prognostic value of increased uptake in SPECT/CT in asymptomatic wrists.

Methods: Forty-four patients (18 women, 26 men, median age 42.5 years, range 18-62 years) referred for bone SPECT/CT for painful symptomatic wrist (SW) were additionally imaged on their asymptomatic contralateral wrist (AW). Planar and SPECT/CT images of the SW and AW were performed between July 2014 and September 2016 and retrospectively evaluated for presence, localization, intensity and origin of uptake using a 4-point grading scale ( $0=$ no uptake, $1=$ low uptake, 2 = moderate uptake, 3 = high uptake). Fourteen patients with increased uptake in the AW were available for clinical follow-up (median follow-up: 11 months, range 3-15 months).

Results: Thirty-one (70.5\%, 95\% confidence interval (Cl): [54.8\%, 83.2\%]) patients showed increased uptake in the SW and $14(31.8 \%, 95 \% \mathrm{Cl}:[18.6 \%, 47.6 \%])$ in the contralateral AW. Mean maximum uptake grade in the SW was 1.48 (range 0-3) and 0.48 (range $0-2$ ) in the AW, respectively. The SW showed significantly more locations with increased uptake $(p<0.001)$ and significantly higher maximum uptake grades compared to the AW $(p<0.001)$.

Abnormal uptake in AW was due to osteoarthritis $(n=7)$, mechanical overload $(n=5)$, trauma $(n=1)$ and a normal variation of an ankylosis $(n=1)$. No patient of the follow-up group developed spontaneous pain in the primarily AW with the exception of 5 patients where wrist examination triggered local pain in the anatomical region of increased SPECT/CT uptake.
\end{abstract}

Conclusions: One third of the contralateral asymptomatic wrists demonstrate low to intermediate grade uptake in bone SPECT/CT. The majority of uptake in the contralateral asymptomatic wrist remained clinically silent in the short time follow-up.

Keywords: SPECT/CT, Wrist, Asymptomatic, Bone scintigraphy

\section{Introduction}

Wrist pain is frequent and attributable to a diversity of diseases (Ciresi and Kerley 1993; Huellner et al. 2013; Pin et al. 1990). Depending on the symptoms, conventional x-rays are the first line imaging modality for the evaluation of wrist pain. Fractures,

(c) The Author(s). 2018 Open Access This article is distributed under the terms of the Creative Commons Attribution 4.0 International License (http://creativecommons.org/licenses/by/4.0/), which permits unrestricted use, distribution, and reproduction in any medium, provided you give appropriate credit to the original author(s) and the source, provide a link to the Creative Commons license, and indicate if changes were made. 
osteoarthritis and inflammatory changes are normally correctly diagnosed with $\mathrm{x}$-rays or ultrasound. In patients where conventional imaging remains insufficient to depict the etiology of the complaints, MR, CT or conventional bone scintigraphy are commonly performed depending on the suspected diagnosis and availability of the different imaging modality. SPECT/CT (Single photon emission computed tomography/computed tomography) is increasingly used as a surrogate to conventional planar scintigraphic images, especially in pathologies involving complex anatomic regions like spine, wrist or foot (Buck et al. 2008; Bybel et al. 2008; Corre et al. 2016; Huellner et al. 2012; Linke et al. 2010; Mariani et al. 2010; Mohan et al. 2010; Nathan et al. 2012; Schleich et al. 2012; Shirley et al. 2016). In symptomatic patients increased uptake in SPECT/CT commonly narrows the differential diagnosis and is often able to pinpoint to the anatomical structure being causative for the patient's symptoms and therefore guide the appropriate treatment. Schleich et al. used SPECT/CT in patients with unspecific wrist pain and described a correlation between the clinical diagnosis and SPECT/CT findings in 30 out of 51 patients (59\%) (Schleich et al. 2012). Scintigraphic uptake in asymptomatic joints and bones with questionable clinical importance is well known in daily routine work and might lead to overdiagnosis and overtreatment. Therefore the aim of this study is to evaluate the prevalence and prognostic value of abnormal uptake in bone SPECT/CT in asymptomatic wrists.

\section{Material and methods}

Patients

Forty-four patients (18 women, 26 men, median age 42.5 years, range 18-62 years) were referred for bone scintigraphy with SPECT/CT by dedicated wrist surgeons following a detailed clinical physical examination of both wrists. Eighty-eight wrists were divided into symptomatic ipsilateral wrists (SW) and asymptomatic contralateral wrist (AW). Main clinical indication for SPECT/CT imaging was as follows: chronic pain after trauma or suggested osteoarthritis in the symptomatic wrist/hand. Approval of the Ethics Committee was obtained for this study (EKNZ Nr: 2016-01647).

\section{SPECT/CT imaging}

Dual phase planar and SPECT/CT images of the symptomatic and asymptomatic wrists were obtained between July 2014 and September 2016. In all patients, bone scintigraphy using ${ }^{99 m} \mathrm{Tc}-3,3-$ diphosphono-1,2-propanedicarboxylic acid $\quad\left({ }^{99 \mathrm{~m}} \mathrm{Tc}-\mathrm{DPD}\right)$ (Teceos, Behringwerke AG, Marburg) was performed. The radiotracer was injected intravenously (mean activity $676 \mathrm{MBq}$, range 619-719). Early phase planar images of both wrists were obtained 5 min after injection (matrix $256 \times 256$ pixels, field of view: $40 \mathrm{~cm}$ ) with a hybrid SPECT/CT system with a built-in flat panel CT component (BrightView XCT; Philips Healthcare, Best, The Netherlands). Planar late-phase images of both wrists were obtained $3 \mathrm{~h}$ after radiotracer injection (matrix $256 \times 256$ pixels, FOV $40 \mathrm{~cm}$ ). Subsequently, SPECT/CT of the wrists was acquired. Patients were positioned prone with arms outstretched. SPECT was performed in step-and-shoot mode with 64 projections and a frame time of $20 \mathrm{~s}$ (SPECT frame format $128 \times 128,128$ views over $360^{\circ}$, step and shoot noncircular acquisition). CT images were acquired in highresolution mode with an isotropic voxel size of $0.33 \times 0.33 \times 0.33 \mathrm{~mm}$ (CT slice 
thickness $0.33 \mathrm{~mm}$, matrix $512 \times 512 \mathrm{~mm})$ with $30 \mathrm{~mA}(219.3 \mathrm{mAs})$ and $120 \mathrm{kV}$ and reconstructed with iterative filters. SPECT images were reconstructed with an iterative 3-D ordered subsets (4 iterations, 16 subsets) expectation maximization (OSEM) algorithm (Astonish, Philips). In 5 patients SPECT/CT images were performed with additional arthrography like described in previously studies (Strobel et al. 2014). SPECT and CT images were fused automatically with a dedicated software (Extended Brilliance Workspace; Philips Healthcare), reformated in coronal and sagittal plane.

\section{Image evaluation/interpretation}

All images were analysed using the local PACS (Merlin PACS, Phönix- PACS, Freiburg, Germany). Images were evaluated by a dual board certified nuclear medicine physician and radiologist with more than 10 years experience in musculoskeletal SPECT/CT reading. The reader was blinded regarding the clinical information and painful wrist. Presence, localization, intensity of radionuclide uptake was assessed using a 4-point grading scale $(0=$ no uptake; $1=$ low uptake $=$ uptake slightly higher than in the normal bone, $2=$ moderate uptake $=$ uptake clearly higher than in the normal bone, $3=$ high uptake = uptake strongly higher than in the normal bone). Additionally, abnormal CT findings without radionuclide uptake were assessed. Abnormal radionuclide uptake was categorised into the following categories: osteoarthritis, mechanical overload, inflammation, traumatic and others.

\section{Statistics}

The statistical analysis were performed using Stata (version 14.1, StataCorp, College Station, Texas, USA). Differences in the number of localizations and the uptake grades were compared between the symptomatic and asymptomatic wrists utilizing frequency tables, descriptive statistics and Wilcoxon signed-rank tests. Proportions were given with 95\% Clopper-Pearson confidence intervals.

\section{Clinical follow-up}

Patients with increased uptake $(n=14)$ in the asymptomatic wrist were subsequently invited for a clinical follow-up exam. Clinical follow up was performed after a median time interval of 9.9 months and a time range of 3-15 months. Clinical examination included assessment of patient's handedness, profession, pain and previous operations or traumas. A detailed clinical examination was performed by the head of wrist surgery who was previously informed about the results of the initial radionuclide uptake in SPECT/CT imaging. Range of movement was tested, stability of the wrist was checked and focused compression in the area of increased uptake in the SPECT/CT images was performed to identify possible painful trigger points.

\section{Results}

Increased early phase uptake was found in 7 (15.9\%, 95\% CI: [6.6\%, 30.1\%]) SW and only in $1(2.3 \%, 95 \%$ CI: [0.0\%, 12.0\%]) AW. 31 (70.5\%, 95\% CI: [54.8\%, 83.2\%]) SW showed increased uptake in late phase SPECT/CT. Fourteen (31.8\%, 95\% CI: [18.6\%, 47.6\%]) AW showed increased uptake in late phase SPECT/CT. The mean number of locations with increased uptakes in AW was 0.55 (range 0- 
4) and 1.27 (range 0-4) in SW (detailed distribution is given in Fig. 1). In the SW there were significantly more locations with abnormal uptake compared with the AW ( $p<0.001$, Wilcoxon signed-rank test). Mean maximum uptake grade in the SW was 1.48 (range $0-3$ ) and 0.48 (range $0-2$ ) in the AW. Of the 14 patients with uptake in the AW 6 patients had uptake in the left wrist (1 of them left-handed) and 8 in the right wrist (7 of them right-handed). In the SW there were 14 wrists with grade 3 uptake, 6 with grade 2 and 11 with grade 1 uptake in late phase $\mathrm{SPECT} / \mathrm{CT}$. In the AW there was no wrist with grade 3 uptake, 7 with grade 2 and 7 with grade 1 uptake (Figs. 2, 3, 4 and 5). Significantly higher uptake grades were observed in the SW group compared to the AW group $(p<0.001$, Wilcoxon signed-rank test).

Radionuclide uptake in the different locations in the SW and the AW are listed in Table 1.

In the SW abnormal uptake was related to osteoarthritis $(n=13)$, incipient osteoarthritis $(n=5)$ mechanical overload $(n=14)$, carpal boss $(n=4)$ or other etiologies $(n=7)$. In the AW abnormal uptake was classified as osteoarthritis $(n=7)$, mechanical overload $(n=5)$ or traumatic $(n=1)$. In 1 asymptomatic wrist a normal variation of an ankylosis between lunate and triquetrum bone was diagnosed in the CT without abnormal uptake.

Median age of the patients with uptake in the asymptomatic wrist classified as osteoarthritis was significantly higher $(p=.03)$ with 47 years compared to 35 years of patients without osteoarthritis (mean age 50.1 years vs. 38.5 years).

\section{Clinical follow-up}

Ten of 14 patients with increased uptake in the AW were available for detailed history and clinical follow-up examination. Four patients were only available for their follow-up interview by phone. No patient of the follow-up group developed spontaneous or stress related pain in the primarily asymptomatic wrist and in none

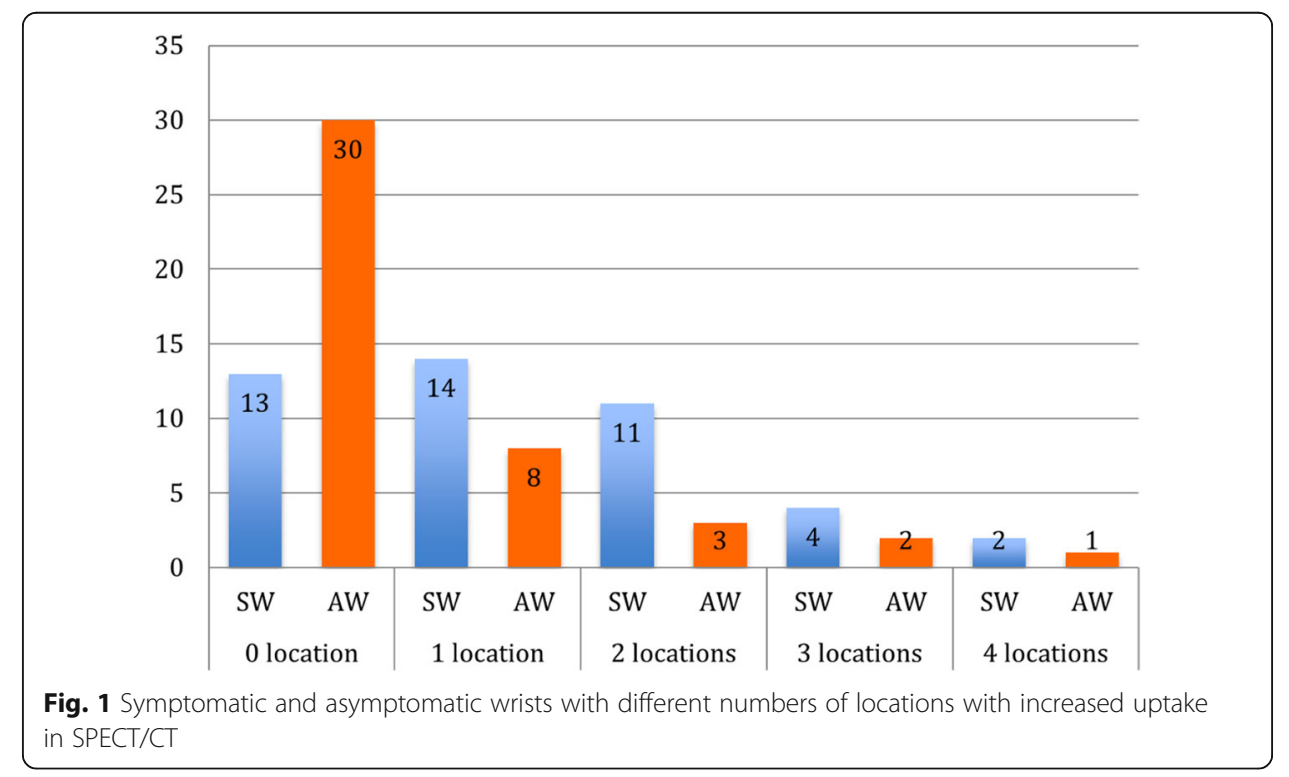




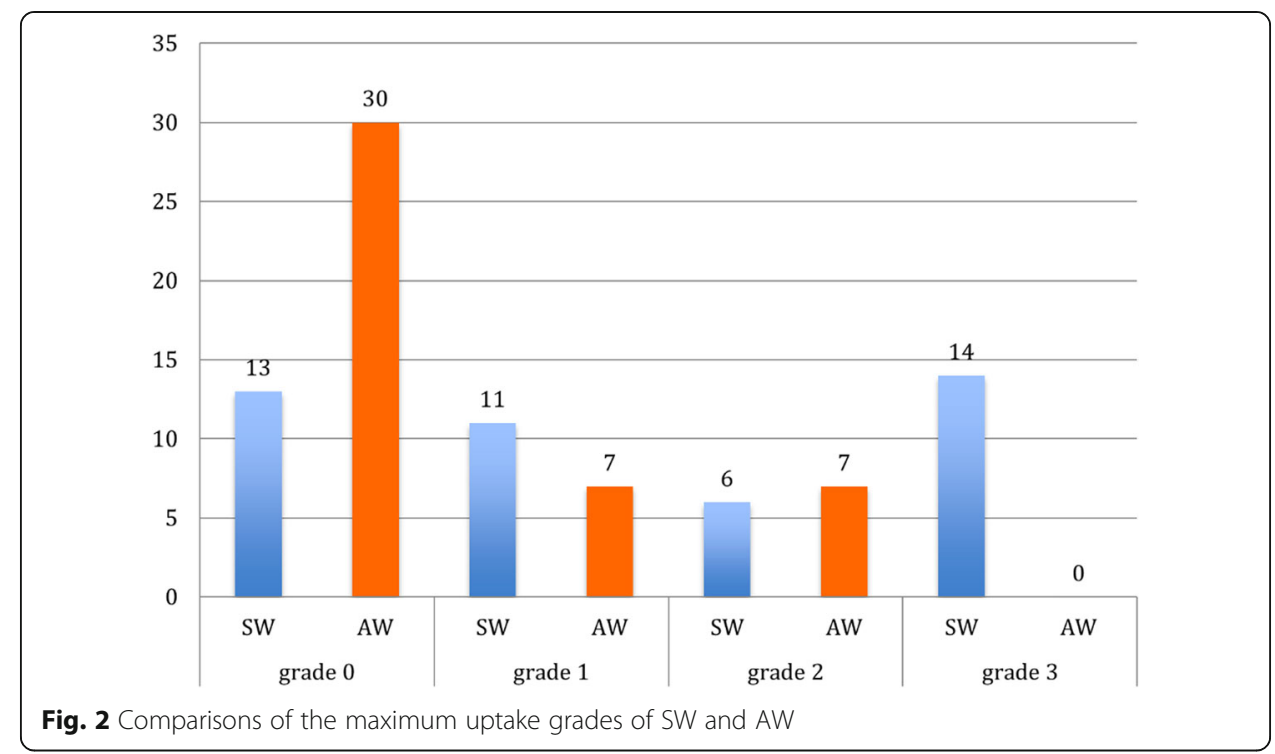

of these patients a specific therapy was necessary during follow-up. One patient mentioned a decrease in physical stress after operation, but no pain. Two patients of the follow-up group had previous operations in the asymptomatic wrist. Three patients had a trauma many years ago. No instability was observed during stress testing. In 5 of 10 asymptomatic wrists focused palpation provoked pain in the region of primarily increased SPECT/CT tracer uptake. Localisations of the provoked pain where the following: CMC I, MCP I/II, pisotriquetral, between capitate and lunate and between hamate and triquetrum. Nine of our 14 patients with asymptomatic wrist uptake reported professions with intense manual daily work.
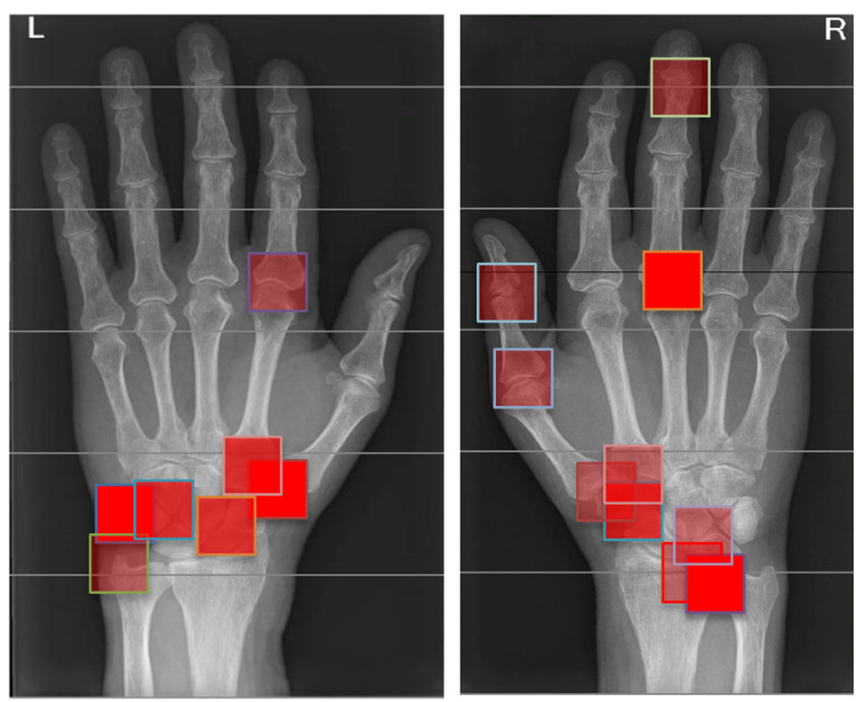

arade 2

$\square$ grade 1

Fig. 3 Summary of number and localizations of increased tracer uptake in the AW split up in the right and left hand of the total 14 patients with tracer uptake. The increasing number of grade of uptake and frequency of cases is represented by the increasing intensity of red color 


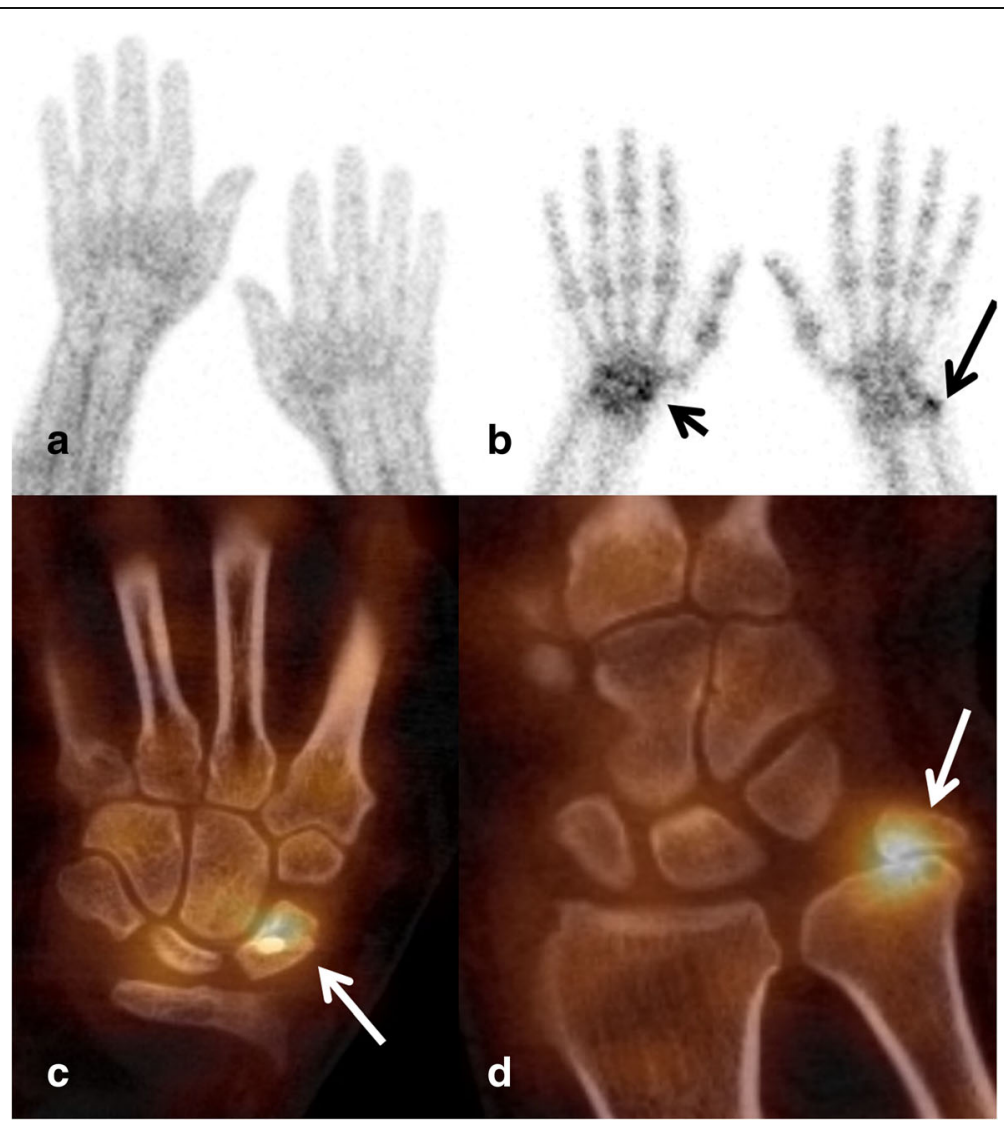

Fig. 4 Thirty-four year old male patient 4 years after reconstruction of a posttraumatic scaphoid non-union with persisting pain and increased uptake in the scaphoid (b short arrow; c long arrow) as well as low grade uptake in all carpal bones in late phase planar images (b) and SPECT/CT (c). No uptake in early phase images (a). The contralateral asymptomatic wrist shows moderate increased uptake in late phase (b long arrow) and SPECT/CT (d long arrow) corresponding to a non-union of the ulnar styloid process with degenerative changes on $\subset$ T. Follow-up clinical investigation 12 months later revealed that the asymptomatic wrist was stable and the range of movement normal. The ulnar styloid fragment was palpable but indolent

\section{Discussion}

The results of this study confirms the presence of increased radionuclide uptake in late phase bone SPECT/CT imaging in approximately one third of asymptomatic wrists. The prevalence and intensity of uptake in asymptomatic wrists was significantly lower compared to symptomatic wrists. In AW only 1 case (2\%) of increased early phase uptake was observed in contrast to $7(16 \%)$ symptomatic patients with increased early phase uptake. This confirms the understanding that early phase uptake reflects primarily active disorders like active inflammation, osteoarthritis or traumatic lesions which are prone to be symptomatic and often require therapy. In contrast incidentally observed late phase SPECT/CT bone uptake in AW showed no diagnostic or therapeutic impact during follow-up time of our patients. Similar observations where previously stated in the literature: Bhure et al. demonstrated the correlation of morphology, SPECT/CT uptake and symptoms in 24 wrists of 21 patients with suspected carpal boss and the considerable number of wrists had discordant findings: absence of local pain with low grade scintigraphic uptake was observed in 3 wrists and moderate uptake in one wrist; local pain without scintigraphic uptake was seen in 6 wrists (Bhure et al. 


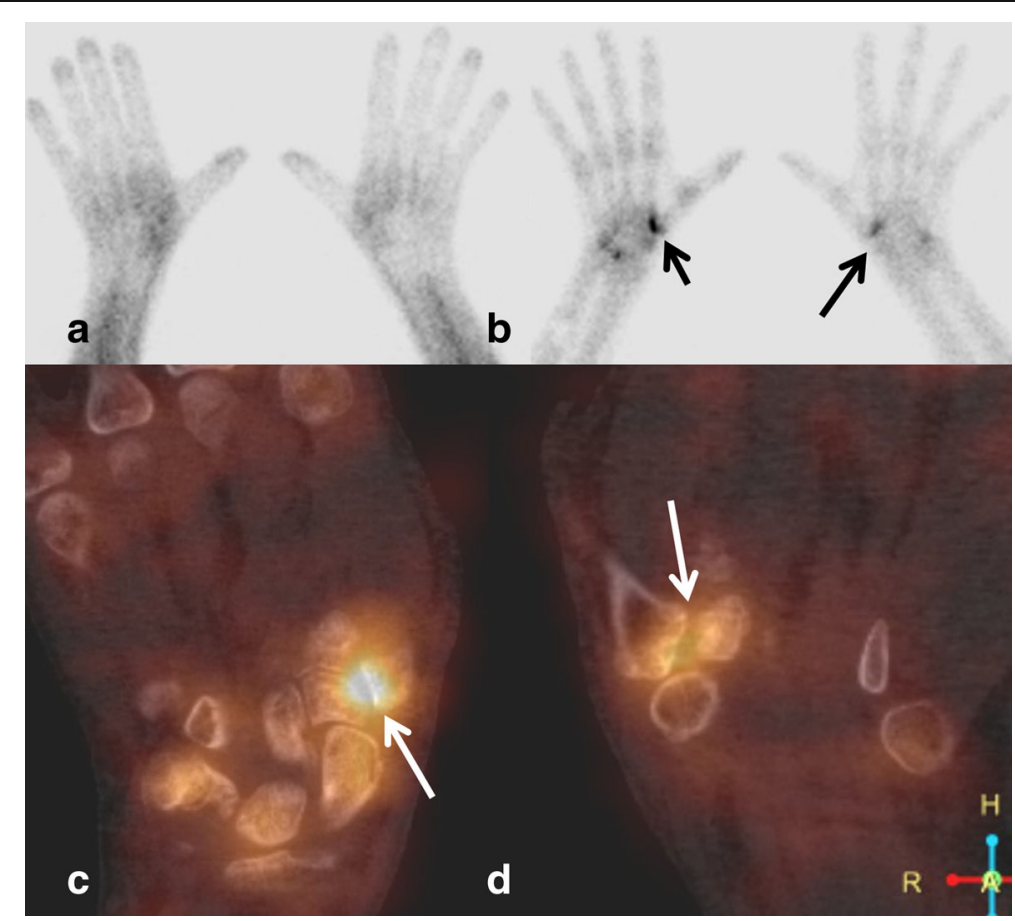

Fig. 5 Planar early phase (a), late phase (b) and SPECT/CT images of the right symptomatic (c) and left asymptomatic (d) wrist in a 59 year old female patient. On the right side trapezectomy and arthroplasty was performed 8 months before with persisting pain and increased late phase uptake in planar late phase (b short arrow) and SPECT/CT (c long arrow) beween trapezoid and base of first metacarpal bone. On the left side trapezectomy was performed 7 years ago and increased low grade uptake (arrow) is present between base of MC 1 and trapezoid bone without any symptoms. In the clinical examination 8 months after SPECT/CT examination the asymptomatic wrist is stable, range of movement normal but pain could be triggered by palpation on the exact localisation on base of metacarpal I and pisotriquetral

2015). Chong et al. recently investigated bone tracer uptake in the accessory navicular bone patients and observed that $8(27 \%)$ patients had grade 1 and 2 patients (3\%) grade 2 uptake (Chong et al. 2016). These results with a prevalence of approximately one third of increased uptake in the foot in asymptomatic patients were in line with our findings in the wrist. Moreover, Avengen et al. investigated patients with knee arthroplasties and found, in general, higher SPECT/CT uptake in symptomatic compared to asymptomatic knees (Awengen et al. 2016). Accordingly, increased radionuclide uptake was also observed in asymptomatic patients. The finding in our study that patients with asymptomatic uptake due to osteoarthritis where significantly older than patients without osteoarthritic uptake supports the theory that joint degeneration increases with age and is often asymptomatic because of missing active inflammation. We know from the large Framingham study with over 1000 participants that about $13.3 \%$ of older men and $26.2 \%$ of older women have at least one hand joint affected by symptomatic osteoarthritis (Zhang et al. 2002). Interestingly, the prevalence of osteoarthritis of at least one affected joint (symptomatic and asymptomatic) on hand $\mathrm{x}$-rays was extremely high (88,6\% in men and $94.4 \%$ in women), showing that the correlation of morphologic osteoarthritis with clinical symptoms of the hand/wrist is even worse than correlation of scintigraphic uptake with clinical symptoms. Over the age of 70 years, approximately $5 \%$ of women and 3\% of men have symptomatic osteoarthritis of the first carpometacarpal joint. There are many different factors contributing to pain in osteoarthritis, 
Table 1 Summary of locations of joint and bone uptake in AW and SW

\begin{tabular}{llll}
\hline Locations of uptake in AW & Number & Locations of uptake in SW & Number \\
\hline radiocarpal & $1(2 \%)$ & radiocarpal & $7(16 \%)$ \\
radioscaphoideal & $1(2 \%)$ & radioscaphoidal & $1(2 \%)$ \\
carpometacarpal I & $4(9 \%)$ & hamate & $1(2 \%)$ \\
carpometacarpal II & $2(5 \%)$ & carpometacarpal I-V \\
metacaropophalangeal III & $4(9 \%)$ & metacaropophalangeal I-V & $17(39 \%)$ \\
metacaropophalangeal I & $1(2 \%)$ & proximal interphalangeal II & $1(2 \%)$ \\
distal interphalangeal III & $1(2 \%)$ & ulnar styloid process \\
ulnar styloid process & $1(2 \%)$ & pisotriquetral & $1(2 \%)$ \\
pisotriquetral & $2(5 \%)$ & scapho-trapezoid-trapezium & $2(5 \%)$ \\
scaphoid & $1(2 \%)$ & hamate-capitate & $3(7 \%)$ \\
hamate-triquetrum & $1(2 \%)$ & lunate & $1(2 \%)$ \\
scapho-trapezoid-trapezium & $1(2 \%)$ & distal radioulnar & $2(5 \%)$ \\
capitate-lunate & $1(2 \%)$ & radio-lunate & $3(7 \%)$ \\
distal radioulnar & $1(2 \%)$ & luno-capitate & $1(2 \%)$ \\
& & scaphoid & $1(2 \%)$ \\
& & scaphoid-capitate & $2(5 \%)$ \\
& & ulno-carpal & $1(2 \%)$ \\
& & Intermetacarpal & $1(2 \%)$ \\
& & $1(2 \%)$ \\
\hline
\end{tabular}

which are extensively discussed in the article of Hunter et al. in 2008 (Hunter et al. 2008). The two factors, which are probably mainly responsible for increased uptake on bone scans are the associated arthritis/synovitis causing early phase uptake and the damage to the subchondral bone causing late phase uptake. Besides osteoarthritis there are various conditions which might cause increased bone turnover and uptake on bone scan like older trauma, benign bone lesions like enchondroma or osteonecrosis.

SPECT/CT has been proven to be helpful in patients with unspecific wrist pain for the detection of occult fractures and special situations like ulnocarpal impaction (Huellner et al. 2013; Corre et al. 2016; Schleich et al. 2012; Shirley et al. 2016; Strobel et al. 2014; Allainmat et al. 2013). Wrist SPECT/CT is often used as third-line problem solving modality in unclear cases after X-ray and CT or MR imaging. Compared to MR, SPECT/CT is less vulnerable to artefacts caused by metallic implants and is feasible in patients with contraindications for MR imaging. The combination of intraarticular contrast and SPECT/CT, called SPECT/CT arthrography, even enables the visualization of important structures like TFCC (triangular fibrocartilage complex), LT (lunotriquetral), SL (scapholunar) ligaments and cartilage and delivers results comparable to MR arthrography (Strobel et al. 2014; Kruger et al. 2011).

In the late phase images radionuclide uptake was observed in locations of asymptomatic wrists predominantly caused by osteoarthritis or mechanical overload. Heavy physical work is a known factor of osteoarthritis (Schmid et al. 1999). Schmid et al. found typical radiological changes of osteoarthritis in metacarpophalangeal joints in heavily working farmers. In our study we could also observe a positive correlation between patients work history and SPECT/CT findings. Nine of our 14 patients with asymptomatic wrist uptake underwent professions with intense manual work. 
Limitations of our studies may be seen with regard of the relatively short clinical follow-up of a median follow-up time of 9.9 months. It remains unclear whether in a subset of patients with clinically silent wrists but abnormal radionuclide uptake might develop clinical symptoms in a long-term follow-up. This was a retrospective study with limited number of patients available for detailed clinical follow-up.

\section{Conclusion}

Increased uptake in SPECT/CT of asymptomatic wrists can be found in approximately one third of patients and is often low to intermediate grade uptake. The majority of uptake in asymptomatic wrists is clinically irrelevant in a median follow-up time of 9.9 months. Careful correlation of increased uptake with clinical investigation is necessary to avoid overdiagnosis and unnecessary therapeutic interventions.

\section{Acknowledgments}

We thank the team of the chief technician Savo Matijasevic for their great support and realization of SPECT/CT investigations.

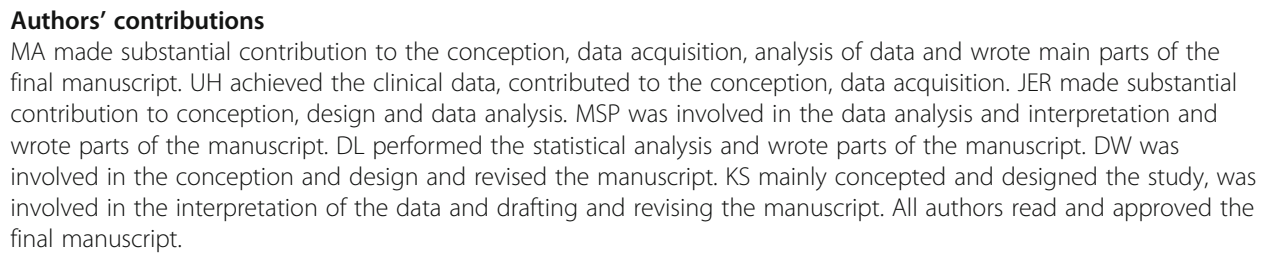

\section{Ethics approval and consent to participate}

All procedures performed in studies involving human participants were in accordance with the ethical standards of the institutional and/or national research committee and with the 1964 Helsinki declaration and its later amendments or comparable ethical standards.

The study was approved by the local ethics committee (Ethikkommission Zentral- und Nordwestschweiz (EKNZ), Registration-Nr: 2016-01647). All procedures performed in this study involving human participants were in accordance with the ethical standards.

Informed consent was obtained from all individual participants who underwent clinical follow-up. Informed consent from the other patients was waived by the ethics committee due to the retrospective design.

\section{Competing interests}

The authors declare that they have no competing interests.

\section{Publisher's Note}

Springer Nature remains neutral with regard to jurisdictional claims in published maps and institutional affiliations.

\section{Author details}

${ }^{1}$ Nuclear Medicine and Radiology, Cantonal Hospital Lucerne, Spitalstrasse, 6000 Luzern 16, Switzerland. ²Division of Hand and Plastic Surgery, Lucerne Cantonal Hospital, 6000 Luzern 16, Switzerland. ${ }^{3}$ Clinic of Radiology and Nuclear Medicine, University Hospital Basel, Spitalstrasse 21, 4056 Basel, Switzerland. ${ }^{4}$ Department of Health Sciences and Health Policy, University of Lucerne, Frohburgstrasse 3, 6002 Luzern, Switzerland.

Received: 2 August 2017 Accepted: 13 December 2017

Published online: 12 February 2018

\section{References}

Allainmat L, Aubault M, Noel V, Baulieu F, Laulan J, Eder V (2013) Use of hybrid SPECT/CT for diagnosis of radiographic occult fractures of the wrist. Clin Nucl Med 38(6):e246-e251. doi: https://doi.org/10.1097/RLU.0b013e318263819e

Awengen R, Rasch H, Amsler F, Hirschmann MT (2016) Symptomatic versus asymptomatic knees after bilateral total knee arthroplasty: what is the difference in SPECT/CT? Eur J Nucl Med Mol Imaging 43(4):762-772. doi: https://doi. org/10.1007/s00259-015-3278-0

Bhure U, Hug U, Huellner MW, Grunig H, Zander A, Del Sol Perez Lago M et al (2015) The value of SPECT/CT in carpal boss. Eur J Nucl Med Mol Imaging 42(12):1883-1890. doi: https://doi.org/10.1007/s00259-015-3151-1

Buck AK, Nekolla S, Ziegler S, Beer A, Krause BJ, Herrmann K et al (2008) Spect/Ct. J Nucl Med 49(8):1305-1319. doi: https://doi.org/10.2967/jnumed.107.050195.

Bybel B, Brunken RC, DiFilippo FP, Neumann DR, Wu G, Cerqueira MD (2008) SPECT/CT imaging: clinical utility of an emerging technology. Radiographics 28(4):1097-1113. doi: https://doi.org/10.1148/rg.284075203 
Chong A, Ha JM, Lee JY (2016) Clinical meaning of hot uptake on bone scan in symptomatic accessory navicular bones. Nucl Med Mol Imaging 50(4):322-328. doi: https://doi.org/10.1007/s13139-016-0452-7

Ciresi KF, Kerley SM (1993) Wrist pain. West J Med 158(4):401-402

Corre AL, Huynh KP, Dhaliwal RS, Bain Gl (2016) Development of a protocol for SPECT/CT in the assessment of wrist disorders. J Wrist Surg 5(4):297-305. doi: https://doi.org/10.1055/s-0036-1583314.

Huellner MW, Burkert A, Strobel K, Perez Lago Mdel S, Werner L, Hug U et al (2013) Imaging non-specific wrist pain: interobserver agreement and diagnostic accuracy of SPECT/CT, MRI, CT, bone scan and plain radiographs. PLoS One 8(12):e85359. doi: https://doi.org/10.1371/journal.pone.0085359

Huellner MW, Strobel K, Hug U, von Wartburg U, Veit-Haibach P (2012) SPECT/CT in diagnostics of the hand joint. Radiologe 52(7):621-628. doi: https://doi.org/10.1007/s00117-011-2269-9.

Hunter DJ, McDougall JJ, Keefe FJ (2008) The symptoms of osteoarthritis and the genesis of pain. Rheum Dis Clin N Am 34(3):623-643. doi: https://doi.org/10.1016/j.rdc.2008.05.004

Kruger T, Hug U, Hullner MW, Schleich F, Veit-Haibach P, von Wartburg U et al (2011) SPECT/CT arthrography of the wrist in ulnocarpal impaction syndrome. Eur J Nucl Med Mol Imaging 38(4):792. doi: https://doi.org/10.1007/ s00259-010-1712-x

Linke R, Kuwert T, Uder M, Forst R, Wuest W (2010) Skeletal SPECT/CT of the peripheral extremities. AJR Am J Roentgenol 194(4):W329-W335. doi: https://doi.org/10.2214/AJR.09.3288

Mariani G, Bruselli L, Kuwert T, Kim EE, Flotats A, Israel O et al (2010) A review on the clinical uses of SPECT/CT. Eur J Nucl Med Mol Imaging 37(10):1959-1985. doi: https://doi.org/10.1007/s00259-010-1390-8

Mohan HK, Gnanasegaran G, Vijayanathan S, Fogelman I (2010) SPECT/CT in imaging foot and ankle pathology-the demise of other coregistration techniques. Semin Nucl Med 40(1):41-51. doi: https://doi.org/10.1053/j.semnuclmed. 2009.08.004

Nathan M, Mohan H, Vijayanathan S, Fogelman I, Gnanasegaran G (2012) The role of 99mTc-diphosphonate bone SPECT/CT in the ankle and foot. Nucl Med Commun 33(8):799-807. doi: https://doi.org/10.1097/MNM. Ob013e328355880b

Pin PG, Young VL, Gilula LA, Weeks PM (1990) Wrist pain: a systematic approach to diagnosis. Plast Reconstr Surg 85(1): $42-46$

Schleich FS, Schurch M, Huellner MW, Hug U, von Wartburg U, Strobel K et al (2012) Diagnostic and therapeutic impact of SPECT/CT in patients with unspecific pain of the hand and wrist. EJNMMI Res 2(1):53. doi: https://doi.org/10. 1186/2191-219X-2-53

Schmid L, Dreier D, Muff B, Allgayer B, Schlumpf U (1999) Lebenslange landwirtschaftliche Schwerarbeit und Arthroseentwicklung an der Hand-Eine kasuistische Untersuchung. Z Rheumatol 58(6):345-350. doi: https://doi.org/ $10.1007 /$ s003930050193

Shirley RA, Dhawan RT, Rodrigues JN, Evans DM (2016) Bone SPECT-CT: An additional diagnostic tool for undiagnosed wrist pain. J Plast Reconstr Aesthet Surg 69(10):1424-1429. doi: https://doi.org/10.1016/j.bjps.2016.04.011

Strobel K, Steurer-Dober I, Da Silva AJ, Huellner MW, del Sol Perez Lago M, Bodmer E et al (2014) Feasibility and preliminary results of SPECT/CT arthrography of the wrist in comparison with MR arthrography in patients with suspected ulnocarpal impaction. Eur J Nucl Med Mol Imaging 41(3):548-555. doi: https://doi.org/10.1007/s00259013-2584-7.

Zhang Y, Niu J, Kelly-Hayes M, Chaisson CE, Aliabadi P, Felson DT (2002) Prevalence of symptomatic hand osteoarthritis and its impact on functional status among the elderly: the Framingham study. Am J Epidemiol 156(11):1021-1027

\section{Submit your manuscript to a SpringerOpen ${ }^{\circ}$ journal and benefit from:}

- Convenient online submission

- Rigorous peer review

- Open access: articles freely available online

- High visibility within the field

- Retaining the copyright to your article

Submit your next manuscript at $\gg$ springeropen.com 